\title{
MR Myelography for Identification of Spinal CSF Leak in Spontaneous Intracranial Hypotension
}

\author{
J.L. Chazen, J.F. Talbott, J.E. Lantos, and W.P. Dillon
}

\begin{abstract}
BACKGROUND AND PURPOSE: CT myelography has historically been the test of choice for localization of CSF fistula in patients with spontaneous intracranial hypotension. This study evaluates the additional benefits of intrathecal gadolinium MR myelography in the detection of CSF leak.
\end{abstract}

MATERIALS AND METHODS: We performed a retrospective review of patients with spontaneous intracranial hypotension who underwent CT myelography followed by intrathecal gadolinium MR myelography. All patients received intrathecal iodine and off-label gadolinium-based contrast followed by immediate CT myelography and subsequent intrathecal gadolinium MR myelography with multiplanar $\mathrm{T}$ fat-suppressed sequences. CT myelography and intrathecal gadolinium MR myelography images were reviewed by an experienced neuroradiologist to determine the presence of CSF leak. Patient records were reviewed for demographic data and adverse events following the procedure.

RESULTS: Twenty-four patients met both imaging and clinical criteria for spontaneous intracranial hypotension and underwent CT myelography followed by intrathecal gadolinium MR myelography. In 3/24 patients (13\%), a CSF leak was demonstrated on both CT myelography and intrathecal gadolinium MR myelography, and in 9/24 patients (38\%), a CSF leak was seen on intrathecal gadolinium MR myelography $(P=.011)$. Four of 6 leaks identified independently by intrathecal gadolinium MR myelography related to meningeal diverticula. CT myelography did not identify any leaks independently. There were no reported adverse events.

CONCLUSIONS: Present data demonstrate a higher rate of leak detection with intrathecal gadolinium MR myelography when investigating CSF leaks in our cohort of patients with spontaneous intracranial hypotension. Although intrathecal gadolinium is an FDA off-label use, all patients tolerated the medication without evidence of complications. Our data suggest that intrathecal gadolinium MR myelography is a well-tolerated examination with significant benefit in the evaluation of CSF leak, particularly for patients with leak related to meningeal diverticula.

ABBREVIATIONS: $\mathrm{CTM}=\mathrm{CT}$ myelography; $\mathrm{MRM}=$ intrathecal gadolinium MR myelography; $\mathrm{SIH}=$ spontaneous intracranial hypotension

$\mathbf{S}$ pontaneous intracranial hypotension ( $\mathrm{SIH})$ is a debilitating condition with protean symptoms, which is often misdiagnosed at initial presentation. ${ }^{1,2}$ The most common cause of SIH is a spinal CSF leak. Patients often have an underlying connective tissue disorders, though underproduction or increased absorption of CSF, dural elasticity, and minor trauma, including disk

Received January 9, 2014; accepted after revision March 3.

From the Department of Radiology (J.L.C., J.E.L.), Weill Cornell Medical Center, New York, New York: Department of Radiology (J.F.T.), San Francisco General Hospital, San Francisco, California; and Department of Radiology and Biomedical Imaging (W.P.D.), University of California, San Francisco, San Francisco, California.

Please address correspondence to J. Levi Chazen, MD, Department of Radiology, Weill Cornell Medical Center, 525 East 68th St, Box 141, New York, NY 10065; e-mail: jlc2008@med.cornell.edu

http://dx.doi.org/10.3174/ajnr.A3975 herniation, may all be contributing factors. ${ }^{3,4}$ A reduction in CSF volume leads to compensatory dilation of venous structures in the brain, which may result in headache and subdural collections via meningeal traction. ${ }^{5,6}$ Severe untreated cases of SIH can lead to coma and stroke. ${ }^{7}$

CT myelography (CTM) has historically been considered the study of choice for the detection and localization of CSF leak, though a criterion standard test is difficult to establish, given the varied etiologies for $\mathrm{SIH}^{8}$ Recent literature has questioned whether CTM is the most sensitive technique for the detection and localization of CSF leaks. ${ }^{9,10}$ More recent techniques have been described, including dynamic CTM, digital subtraction myelography, heavily T2-weighted spinal MR imaging, and intrathecal gadolinium MR myelography (MRM). Both dynamic CT and digital subtraction myelography have been advocated in cases 
Leak Detection by Modality

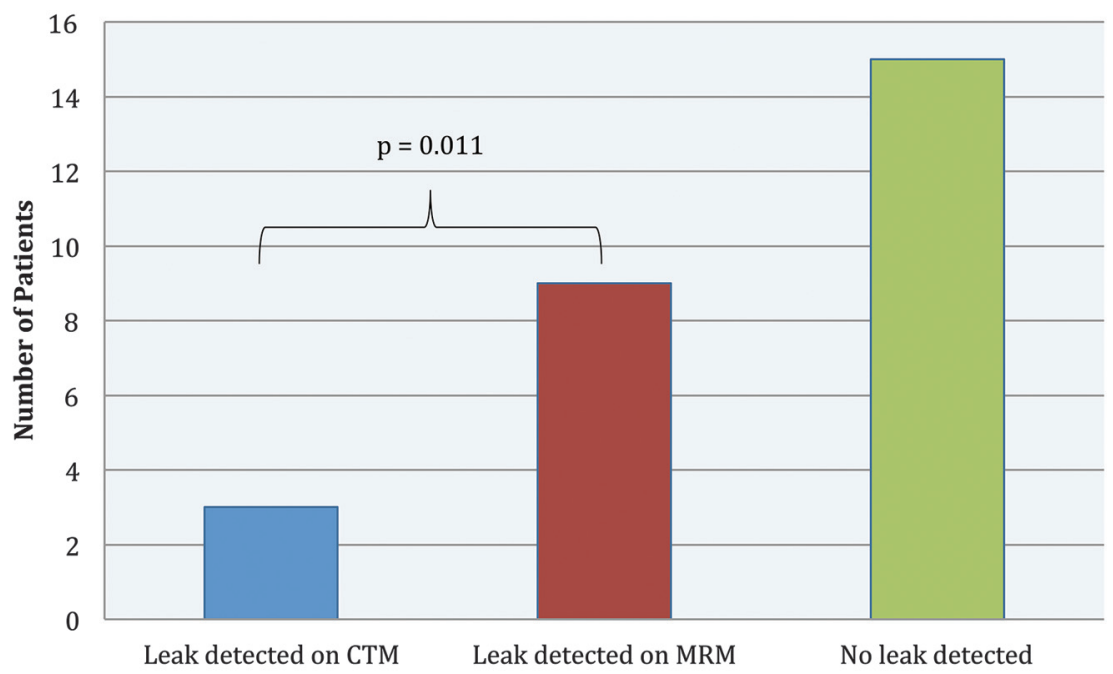

FIG 1. Bar graph illustrating the rate of leak detection by CTM and MRM. A statistically significant difference was observed between CTM and MRM by a 2-tailed paired $t$ test $(P=.011)$.
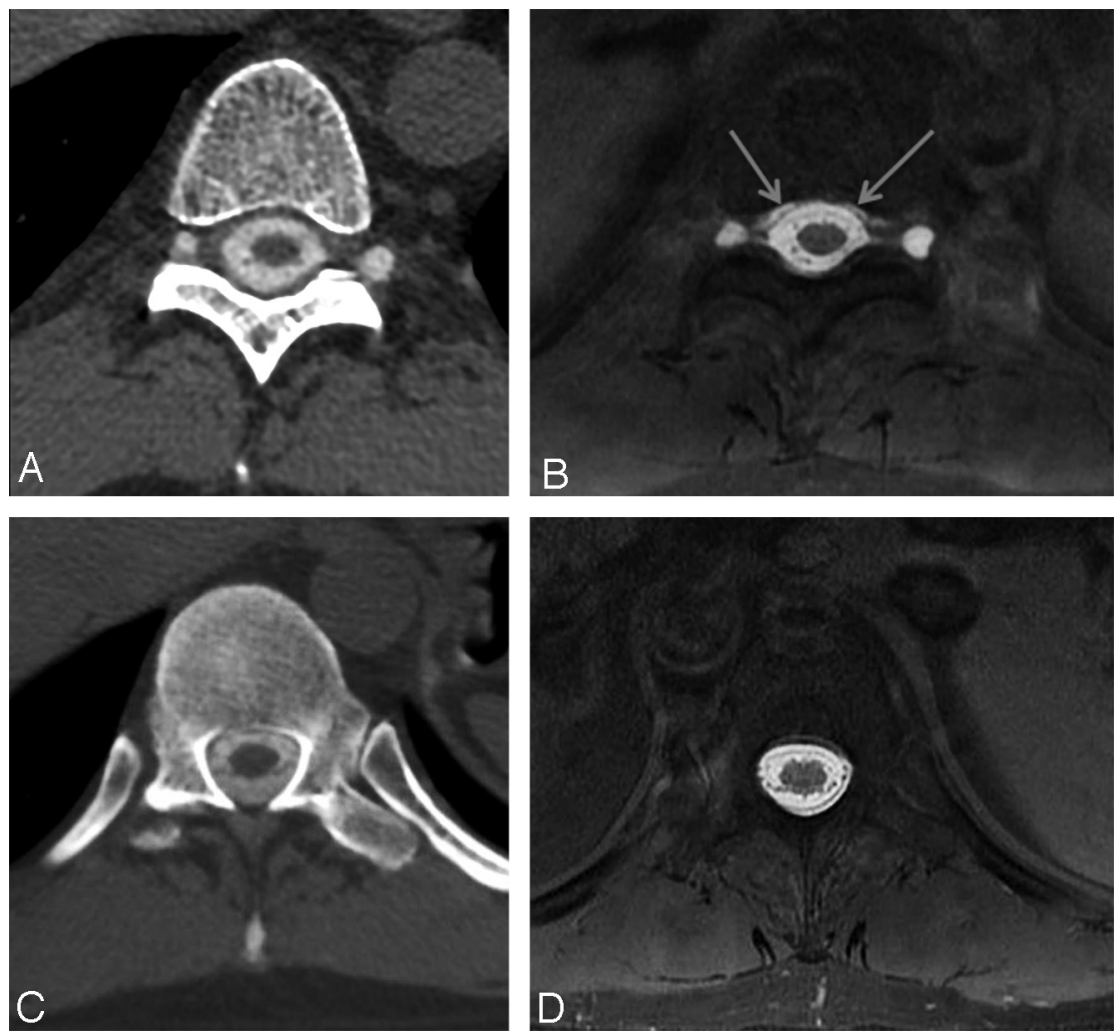

FIG 2. Patient 4. A 52-year-old former fire fighter with a 1-year history of postural headaches. Axial CTM $(A$ and $C)$ and axial T1 fat-suppressed images from MRM ( $B$ and $D)$ following intrathecal administration of iodinated contrast and gadolinium. Multiple spinal diverticula are seen along with an extradural contrast collection (arrows, B) not evident on a concurrent CTM examination.

with significant spinal extra-arachnoid fluid collections on preprocedural spinal MR imaging. ${ }^{11} \mathrm{MRM}$ with intrathecal gadolinium has been shown to have a high rate of leak detection and appears safe in small doses used for myelography. ${ }^{12}$

The mainstay of treatment for SIH is autologous epidural blood patch, initially effective in about one-third of patients. ${ }^{13}$
Directed epidural blood patch at the site of CSF fistula with CT guidance has been shown to be effective. ${ }^{14}$ Targeted therapy may improve clinical outcomes with evidence of benefit when the blood patch is performed as close as possible to the site of CSF fistula. ${ }^{13}$ Therefore, diagnostic techniques that precisely localize a CSF leak are important for guiding therapy, particularly for treatments such as fibrin glue injection and surgical repair. $^{2}$

Our study evaluates the relative benefit of intrathecal gadolinium MRM compared with CTM in detecting and localizing CSF leaks in patients with previously confounding diagnostic work-up to guide treatment in patients with SIH.

\section{MATERIALS AND METHODS}

A waiver of informed consent was obtained from our Committee on $\mathrm{Hu}$ man Research for this retrospective review, consistent with the Health Insurance Portability and Accountability Act. A search of the radiology information system between May 2007 and April 2013 yielded 30 patients who had lumbar puncture and intrathecal instillation of iodine- and gadoliniumbased contrast followed by CTM and subsequent MRM. Many patients in our cohort had prior unrevealing diagnostic work-up for symptoms of SIH and were referred to our institution for further evaluation.

All patients with contrast brain MRI as well as CTM and MRM studies were reviewed for evidence of SIH by a single attending neuroradiologist (W.P.D.) with $>20$ years of experience and a Certificate of Added Qualification in neuroradiology. Patients were included if they had clinical and imaging evidence of $\mathrm{SIH}$, and all patients met the modified criteria of Schievink et $\mathrm{al}^{15}$ for SIH, including orthostatic headache, brain MR imaging with pachymeningeal enhancement and/or brain sagging, and absence of recent lumbar puncture or other attributable intervention. Multiplanar images from CTM and MRM were reviewed to determine whether a CSF leak was present. A CSF leak on CTM or MRM was defined as an extra-arachnoid collection of contrast unrelated to the needle puncture. Patients were excluded if they did not meet the modified criteria of Schievink et al for SIH or if complete demographic or imaging information was unavailable. 
A combined CTM and MRM imaging procedure was performed as follows: Informed consent was obtained after the discussion of off-label use of gadolinium in the intrathecal space. All studies were performed by using a CT-guided lumbar puncture with a 22-ga spinal needle. Opening pressure was measured in the decubitus position. A mixture of 10-mL Omnipaque (iohexol, $240 \mathrm{mg} / \mathrm{mL}$; GE Healthcare, Princeton, New Jersey) and 0.3-mL Magnevist (gadopentetate dimeglumine, $0.5 \mathrm{~mol} / \mathrm{L}$; Schering, Berlin, Germany) was then instilled intrathecally, and the spinal needle was removed. After repositioning the patient on a gurney to facilitate passage of contrast throughout the spinal canal, including Trendelenburg positioning, the patient was transferred back to the CT scanner table for CTM.

CT of the spinal axis was performed on a LightSpeed VCT 64-detector row scanner (GE Healthcare, Milwaukee, Wisconsin) with a rotation time of 0.8 seconds, detector coverage of $40 \mathrm{~mm}$, pitch of $0.98: 1$, section thickness of $0.625 \mathrm{~mm}$, and $100 \mathrm{kV}$ with auto-mA, and noise index of 10.8. Multiplanar reformations were generated for review. The patient was then transferred to the MR imaging suite.

MR imaging of the spinal axis was performed on a 3T Discovery MR imaging scanner (GE Healthcare) an average of 45 minutes following the CT myelogram. T1-weighted sagittal, coronal, and axial imaging was acquired by a using modified 3-point Dixon fat-suppression technique at 4-mm section thickness with no skip. We used the following MR imaging parameters: TR, 400-750 ms; echo train length, 3-5; bandwidth, $50 \mathrm{~Hz}$; TE, $10-50 \mathrm{~ms}$ ("minimum full" was selected by the technologist depending on TR and echo train length). FOV for axial imaging was $16 \mathrm{~cm}$; for sagittal thoracic and lumbar imaging, $24 \mathrm{~cm}$; and for sagittal cervical imaging, $20 \mathrm{~cm}$. Matrix size was $320-$ 384 in the frequency-encoding direction and 192-256 in the phase direction.

Excel for Mac 2011, Version 14.0 (Microsoft, Redmond, Washington) was used for all statistical analyses. Categoric variables are presented as frequencies and percentages, while continuous variables are presented as means and SDs. The difference in leak detection between CTM and MRM was calculated by using a 2 -tailed paired $t$ test of the means, with significance set at $P<.05$. A 2-tailed test was chosen because the null hypothesis was that neither CTM nor MRM was the more sensitive test; if either had been more sensitive, the null hypothesis would be voided. A paired test was chosen because the same patients underwent both studies.

\section{RESULTS}

Thirty patients were identified with CTM and MRM procedures performed during the study period from May 2007 through April 2013. Twenty-four patients met inclusion criteria, including clinical and brain MR imaging findings of SIH. The mean age was 51 years (range, $20-85$ years), and $58 \%$ of patients were male. The mean opening pressure at lumbar puncture was $7.8 \pm 3.9 \mathrm{~cm} \mathrm{H}_{2} 0$ (range, 0-14.5 $\mathrm{cm} \mathrm{H}_{2} 0$ ).

In 15 of 24 patients (63\%), no CSF leak was found on either CTM or MRM. In 3 of 24 patients (13\%), a CSF leak was demonstrated on both CTM and MRM, and in 6 of 24 patients (25\%), a CSF leak was seen only on MRM. MRM (9/24, 38\%) was more sensitive than CTM $(3 / 24,13 \%)$ for the detection of
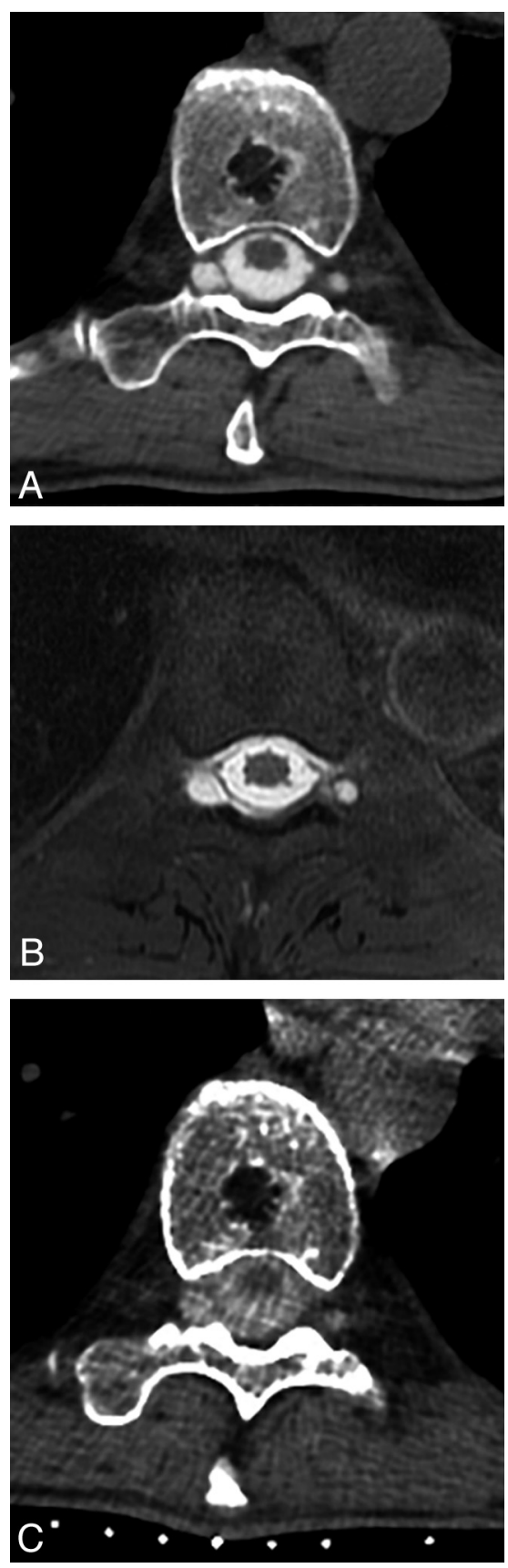

FIG 3. Patient 2. A 62-year-old man with headache and recurrent subdural hemorrhage following evacuation, found to have imaging findings of SIH. Axial CTM $(A)$ and MRM $(B)$ images at the T9-T10 level. Spinal diverticula are evident on both examinations. The MRM demonstrates an extra-arachnoid contrast collection and ill-defined increased $\mathrm{T}$ signal surrounding the enlarged right spinal diverticula. Subsequent CT performed for epidural blood patch planning with a localization grid in place $(C)$ shows the spinal diverticula; the extraarachnoid contrast collection is not evident. The patient reported symptomatic relief following directed blood patch and was without headache as of the 2-month follow-up note.

CSF leak $(P=.011)$. No patients had a leak seen exclusively on CTM (Fig 1).

The MRM examination followed the CTM examination by an average of $45 \pm 21$ minutes. Most leaks involved the midthoracic region with extra-arachnoid collections seen in most (7/9) patients with leaks (Table, Figs 2 and 3). Among the 3 patients with 

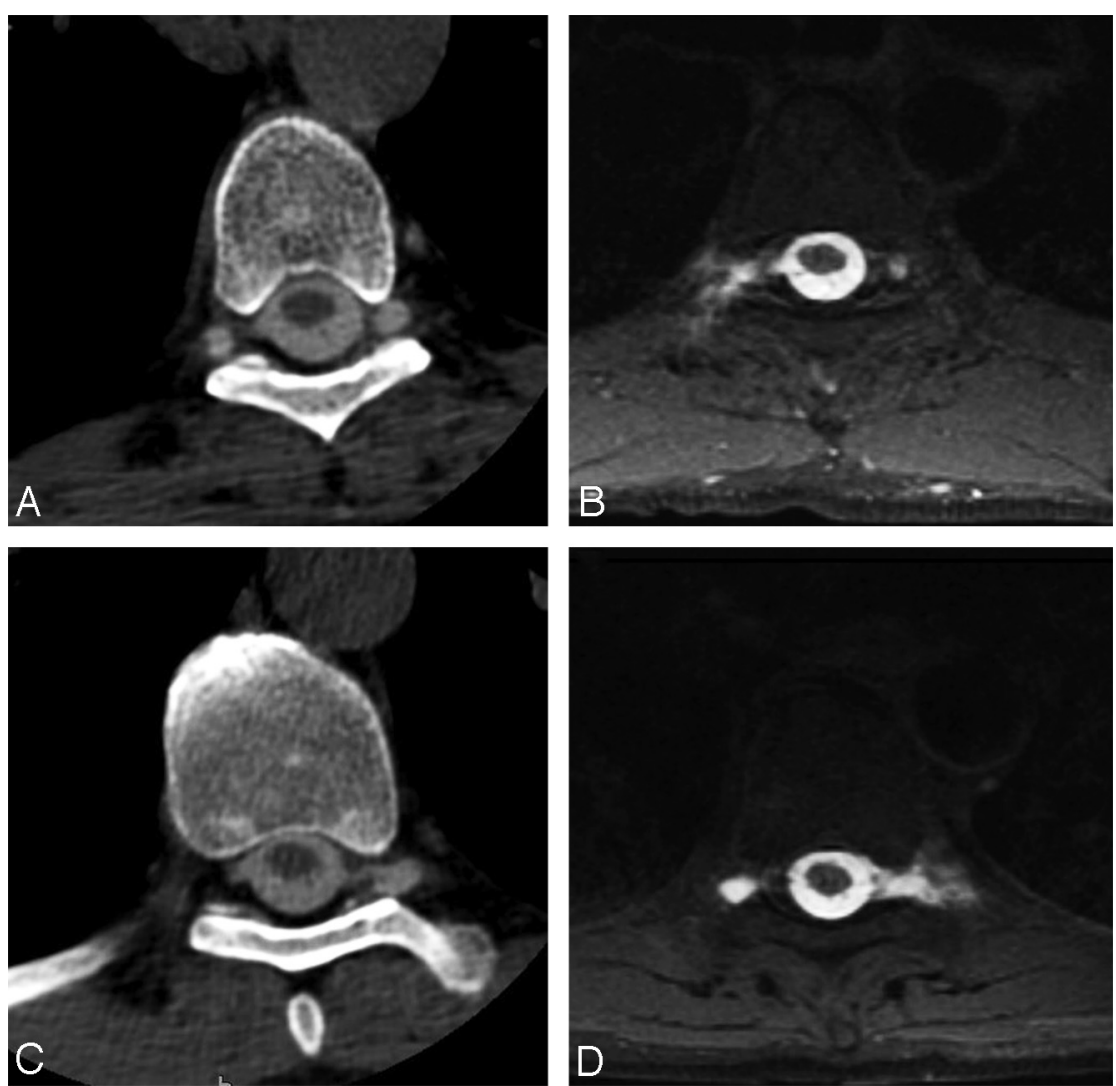

FIG 4. Patient 7. A 55-year-old man with a history of lethargy, fatigue, and hearing loss. Axial CTM $(A$ and $C)$ and axial MRM $(B$ and $D)$ images. $C T$ shows bilateral spinal diverticula. MR image demonstrates prominent contrast leakage from the right-sided T5-T6 (B) and left T10-T11 (D) diverticula, with gadolinium extending into the adjacent paraspinal musculature. The patient underwent targeted epidural blood patches of both leaking spinal diverticula. and guiding treatment for this subpopulation of patients with CSF leak associated with spinal meningeal diverticula.

The sensitivity of CTM for the detection of leaks in patients with clinical symptoms of SIH is difficult to determine given the lack of a robust criterion standard. Patients may demonstrate intracranial hypotension without discrete CSF leak, suggesting that etiologies for the SIH syndrome are varied. In addition to a frank dural tear, SIH may relate to abnormal thecal sac compliance or imbalance between epidural and CSF pressures; ${ }^{17}$ the cause of SIH in patients with negative CTM and MRM findings remains unresolved in most cases. Occult CSF leaks resulting from slow-flow CSF venous fistula may represent an additional etiology for SIH, which would be difficult to identify at imaging, though secondary signs such as early radiotracer within the bladder have been described. Opening pressure is often low but may be within normal limits. ${ }^{18} \mathrm{Un}$ derlying dural and connective tissue abnormalities are hypothesized to contribute to the abnormal CSF egress. ${ }^{19}$ Thus, the potential etiologies for SIH are numerous, and the significant number of patients in our cohort without evidence of leak on multiple imaging examinations

leaks detected on CTM, 2 had a disk herniation likely resulting in a dural tear; the third patient had marfanoid features and leak attributable to dural ectasia. All 6 patients with leaks detected only on MRM had multiple spinal meningeal diverticula (Figs 4 and 5). One patient had a significant epidural collection spanning C3-L1 on MRM and required digital subtraction myelography for leak localization. CT-guided epidural blood patch was performed on all 9 patients with detectable leak and on the same day as CTM and MRM studies when possible (4/9 patients).

\section{DISCUSSION}

MRM was more sensitive than CTM for the detection of CSF leak in our cohort of patients with SIH. A CSF leak was identified in 9 of $24(38 \%)$ patients on MRM but in only 3 of 24 (13\%) patients with CTM $(P=.011)$. CTM did not identify any leaks that were not found on MRM.

Most interesting, 4 of 6 patients in whom a leak was identified on MRM, but not CTM, had leaks in close proximity to spinal meningeal diverticula (Figs 4 and 5). The association between $\mathrm{SIH}$ and spinal meningeal diverticula is well-established. ${ }^{16}$ There is evidence that targeted therapy as close as possible to the site of CSF leak is beneficial; and targeted blood patches, fibrin glue therapy, and surgical ligation of spinal meningeal diverticula have been described with good results. ${ }^{13,14}$ Present data suggest that MRM may be particularly helpful for the detection of CSF leak may reflect slow-flow leaks occult on both CTM and MRM.

We postulate that the overall relatively low rate of leak detection in our cohort is due to patient selection, because many patients in our study were referred to our large tertiary care center when prior outside nuclear or CT localization techniques were unrevealing, thus selecting out patients with more conspicuous leaks. However, our finding of a higher rate of leak detection with MRM compared with CTM is in accordance with recently published studies. ${ }^{11}$ We found a leak at MRM in 9 of 24 patients (38\%) with clinical symptoms and imaging findings of SIH, similar to findings in a recent study by Akbar et al, ${ }^{10}$ which detected a leak on MRM in 5 of 24 patients (21\%) with negative CTM findings. Despite our cohort of diagnostically challenging patients, we recognize the benefit of MRM and advocate MRM examinations early in the diagnostic work-up.

Increased leak detection with MRM over CTM may, in part, be related to the longer imaging half-life of gadolinium in soft tissues. While iodinated contrast will peak and wash out of softtissues rapidly, gadolinium has been shown to maintain sufficient concentrations for T1 shortening for hours after intravenous administration-a property that has been exploited in many fields, including hepatic imaging. ${ }^{20}$ As a result, even though delayed imaging with CTM may still have sufficient contrast inside the subarachnoid space, extradural contrast that has leaked out of the thecal sac may be more difficult to see with CTM than MRM 
because of these different soft-tissue characteristics. It is unclear how the mixture of iodinated contrast with gadolinium affects imaging parameters in our cohort, though there is evidence that iodinated contrast increases T1 and T2 shortening on MR imaging, possibly increasing signal of the contrast mixture on T1 fatsuppressed sequences. ${ }^{21}$

All patients in our study were able to complete both lumbar puncture and subsequent imaging studies. No adverse reactions were observed. In a cohort of 100 patients receiving Gd-DTPA, no adverse events were reported outside of postprocedural headache. ${ }^{22}$ Gd-DTPA has been advocated as the safest agent for in-
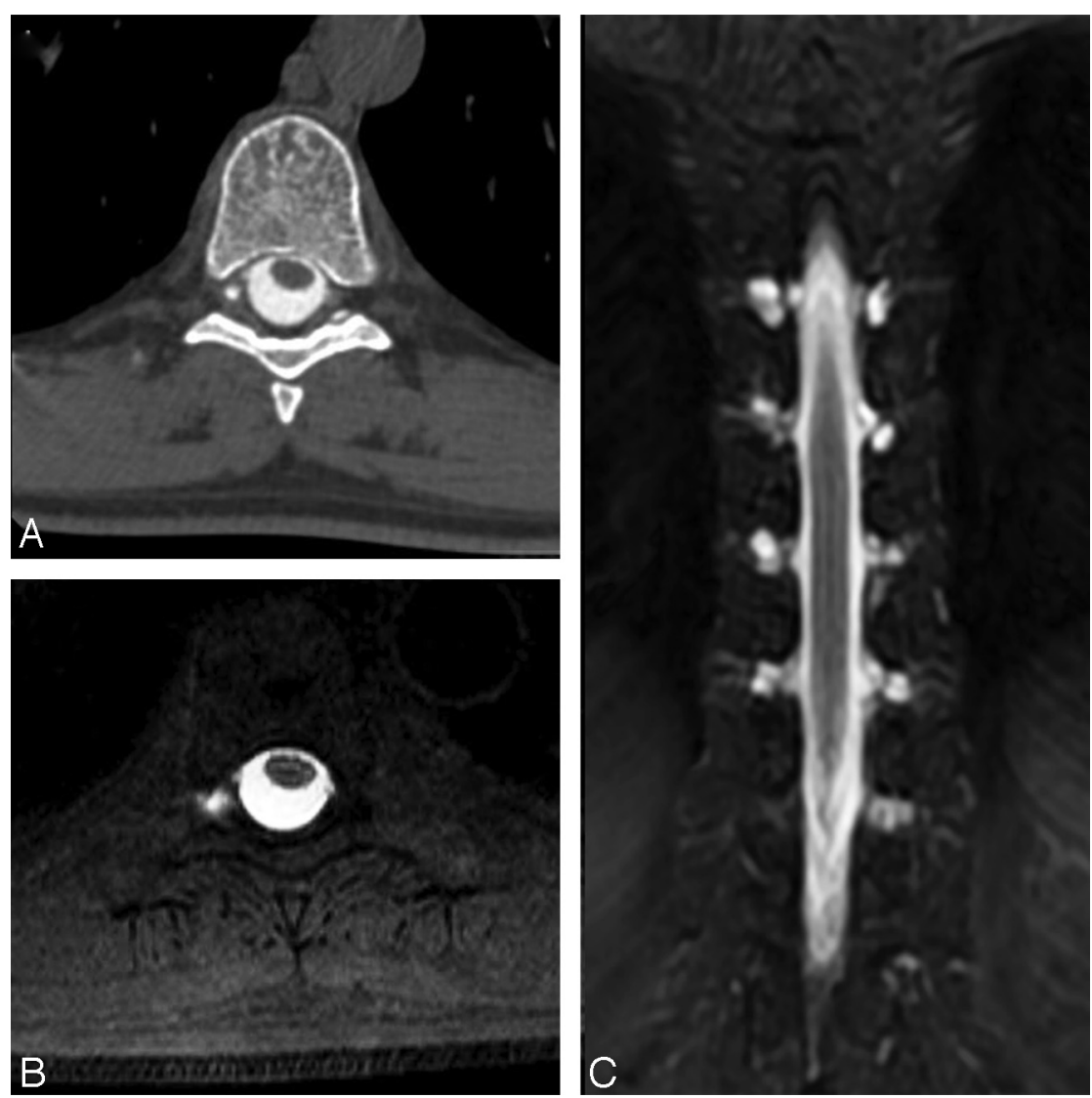

FIG 5. Patient 8. A 55-year-old man with postural headache and low CSF pressure on lumbar puncture. Axial CTM $(A)$, axial MRM (B), and coronal MRM $(C)$ images. Coronal MRM shows multiple spinal diverticula. However, only the right T7-T8 spinal diverticula, seen on the CTM $(A)$, shows evidence of contrast leakage on axial MRM (B), evident by ill-defined T1 shortening surrounding the cyst. This lesion was treated with directed epidural blood patch with symptomatic improvement for 5 years following treatment. trathecal administration. ${ }^{22}$ While there are limited data on the toxic effects of intrathecal gadolinium-based contrast agents, a 2010 report described the onset of adverse symptoms 6 hours following accidental intrathecal gadolinium contrast administration at a dose $20 \times$ that used in our study. ${ }^{23}$ Twenty-four-hour telephone follow-up and subsequent clinical notes did not reveal adverse events attributable to the MRM procedure in our cohort. Despite the apparent safety, physicians need to be mindful that intrathecal gadolinium is an FDA off-label use and should weigh the risks and benefits of the procedure carefully in each patient.

In our experience, CTM and MRM are complementary examinations. While CTM did not independently identify extradural collections, the rapid acquisition following intrathecal contrast administration may provide diagnostic benefits for high-flow leaks and facilitate subsequent targeted treatment. ${ }^{24}$ CTM provides additional anatomic information, for example, bone detail that is better appreciated. Additionally, high-flow spinal CSF leaks may benefit from dynamic CTM because a large-volume leak can obscure a leak site. ${ }^{13}$ Although there are benefits of CTM and MRM performed concurrently, some patients may benefit from MRM performed first followed by CTM, as necessary, to minimize radiation exposure. There is a trade-off with this approach and the possibility of subjecting patients to 2 lumbar puncture procedures.

Limitations of our study include those inherent in a retrospective design and single-center experience with a relatively small number of patients. A possible confounding factor is the delayed nature of MRM in this cohort, which was performed an average of 45 minutes following CTM, likely allowing additional time for contrast to diffuse into small leaks. However, in patients receiving same-day CT-guided blood patches, leaks evident only on the MRM were not

Patients with CSF leak detected on CTM or MRM ${ }^{a}$

\begin{tabular}{|c|c|c|c|c|c|c|c|c|c|}
\hline $\begin{array}{l}\text { Patient } \\
\text { No. }\end{array}$ & $\begin{array}{l}\text { Age } \\
\text { (yr) }\end{array}$ & Sex & $\begin{array}{l}\text { Opening } \\
\text { Pressure } \\
\left(\mathrm{cm} \mathrm{H} \mathrm{H}_{2} \mathrm{O}\right)\end{array}$ & $\begin{array}{c}\text { Leak } \\
\text { Detected } \\
\text { on CTM } \\
\end{array}$ & $\begin{array}{l}\text { Leak Detected } \\
\text { on MR } \\
\text { Myelogram } \\
\end{array}$ & $\begin{array}{c}\text { Time } \\
\text { Difference } \\
\text { (h:min) }\end{array}$ & $\begin{array}{c}\text { Extent of } \\
\text { Extra-Arachnoid } \\
\text { Contrast }\end{array}$ & $\begin{array}{l}\text { Same-Day } \\
\text { Blood } \\
\text { Patch }\end{array}$ & Suspected CSF Leak Etiology \\
\hline 1 & 66 & Female & 5 & No & Yes & $0: 37$ & $\mathrm{~T} 6-\mathrm{T} 12$ & No & Unclear; spinal diverticula C6-C7 through L2-L3 \\
\hline 2 & 62 & Male & 7 & No & Yes & $1: 14$ & T9-L1 & Yes & Bilateral T9-T10 spinal diverticula \\
\hline 3 & 27 & Male & 10 & Yes & Yes & $0: 55$ & L2-L5 & No & Dural ectasia; marfanoid features \\
\hline 4 & 52 & Male & 7 & No & Yes & 1:14 & $\mathrm{T} 6-\mathrm{T} 12$ & Yes & Bilateral T9-T10 and left T11-T12 spinal diverticula \\
\hline 5 & 32 & Female & 0 & No & Yes & $0: 29$ & C3-L1 & No & $\begin{array}{l}\text { No source of leak detected; T2-T3 leak seen on } \\
\text { subsequent fluoroscopic subtraction myelogram }\end{array}$ \\
\hline 6 & 37 & Female & 6 & Yes & Yes & $5: 14$ & T10-L1 & Yes & $\begin{array}{l}\text { T11-T12 disk osteophyte; blood patch performed } \\
\text { before MRM }\end{array}$ \\
\hline 7 & 55 & Male & 8 & No & Yes & $0: 49$ & T5-T6 and T9-T10 & No & Right T5-T6 and left T9-T10 spinal diverticula \\
\hline 8 & 55 & Male & 6 & No & Yes & $0: 12$ & Т7-T8 & Yes & Right T7-T8 spinal diverticula \\
\hline 9 & 58 & Female & 0 & Yes & Yes & $0: 46$ & C7-T4 & No & $\mathrm{T} 1-\mathrm{T} 2$ secondary to disk protrusion and dural tear \\
\hline
\end{tabular}

a The time difference reflects the time stamp difference between CTM acquisition and the beginning of MRM. A same-day blood patch was performed when logistically possible and a clear source of leak was identified. 
seen on delayed CT imaging (Fig 3). Follow-up studies with MRM followed by delayed CTM would be necessary to confirm this observation. This study is also limited by the lack of a criterion standard for leak confirmation. While many of our patients received targeted epidural blood patches with symptomatic improvement, no leaks were confirmed surgically.

\section{CONCLUSIONS}

Our data build on the growing body of evidence that MRM is more sensitive than CTM for the detection of CSF leaks in SIH. ${ }^{5,6}$ This finding is important because treatment with either directed blood patch, fibrin glue injections, or surgery requires preprocedural knowledge of the site of CSF leak. Although intrathecal administration remains an FDA off-label use of gadolinium, all patients in our study tolerated intrathecal gadolinium without complications. MRM is most useful in patients without obvious high-flow leaks who may be better evaluated by dynamic CTM or fluoroscopic myelography. Perhaps most important, MRM was superior to CTM for identifying CSF leaks related to meningeal diverticula. MRM is a well-tolerated examination that appears complementary to CTM with significant benefit in the diagnosis of CSF leaks.

Disclosures: William P. Dillon-UNRELATED: Expert testimony.* Grants/Grants Pending: General Electric, ${ }^{\star}$ Comments: principal investigator on a grant for the evaluation of 4D MR imaging sequences (acted in the place of investigator who was unable to serve as the principal investigator). *Money paid to the institution.

\section{REFERENCES}

1. Renowden SA, Gregory R, Hyman N, et al. Spontaneous intracranial hypotension. J Neurol Neurosurg Psychiatry 1995;59:511-15

2. Schievink WI. Misdiagnosis of spontaneous intracranial hypotension. Arch Neurol 2003;60:1713-18

3. Schievink WI. Spontaneous spinal cerebrospinal fluid leaks and intracranial hypotension. JAMA 2006;295:2286-96

4. Rapport RL, Hillier D, Scearce T, et al. Spontaneous intracranial hypotension from intradural thoracic disc herniation: case report. J Neurosurg 2003;98(3 suppl):282-84

5. Mokri B. Spontaneous cerebrospinal fluid leaks: from intracranial hypotension to cerebrospinal fluid hypovolemia-evolution of a concept. Mayo Clin Proc 1999;74:1113-23

6. George U, Rathore S, Pandian JD, et al. Diffuse pachymeningeal enhancement and subdural and subarachnoid space opacification on delayed postcontrast fluid-attenuated inversion recovery imaging in spontaneous intracranial hypotension: visualizing the Monro-Kellie hypothesis. AJNR Am J Neuroradiol 2011;32:E16

7. Pleasure SJ, Abosch A, Friedman J, et al. Spontaneous intracranial hypotension resulting in stupor caused by diencephalic compression. Neurology 1998;50:18545-47

8. Wang EW, Vandergrift WA 3rd, Schlosser RJ. Spontaneous CSF leaks. Otolaryngol Clin North Am 2011;44:845-56, vii

9. Albes $G$, Weng $H$, Horvath $D$, et al. Detection and treatment of spinal CSF leaks in idiopathic intracranial hypotension. Neuroradiology 2012;54:1367-73

10. Akbar JJ, Leutmer PH, Schwartz KM, et al. The role of MR myelography with intrathecal gadolinium in localization of spinal CSF leaks in patients with spontaneous intracranial hypotension. AJNR Am J Neuroradiol 2012;33:535-40

11. Luetmer PH, Schwartz KM, Eckel LJ, et al. When should I do dynamic CT myelography? Predicting fast CSF leaks in patients with spontaneous intracranial hypotension. AJNR Am J Neuroradiol 2012;33:690-94

12. Albayram S, Kilic F, Ozer H, et al. Gadolinium-enhanced MR cisternography to evaluate dural leaks in intracranial hypotension syndrome. AJNR Am J Neuroradiol 2008;29:116-21

13. Sencakova D, Mokri B, McClelland RL. The efficacy of epidural blood patch in spontaneous CSF leaks. Neurology 2001;57:1921-23

14. Kranz PG, Gray L, Taylor JN. CT-guided epidural blood patching of directly observed or potential leak sites for the targeted treatment of spontaneous intracranial hypotension. AJNR Am J Neuroradiol 2011;32:832-38

15. Schievink WI, Dodick DW, Mokri B, et al. Diagnostic criteria for headache due to spontaneous intracranial hypotension: a perspective. Headache 2011;51:1442-44

16. Medina JH, Abrams K, Falcone S, et al. Spinal imaging findings in spontaneous intracranial hypotension. AJR Am J Roentjenol 2010;195:459-64

17. Franzini A, Messina G, Chiapparini L, et al. Treatment of spontaneous intracranial hypotension: evolution of the therapeutic and diagnostic modalities. Neurol Sci 2013;34(suppl 1):S151-55

18. Mokri B. Spontaneous intracranial hypotension. Curr Pain Headache Rep 2001;5:284-91

19. Grimaldi D, Mea E, Chiapparini L, et al. Spontaneous low cerebrospinal pressure: a mini review. Neurol Sci 2004;25(suppl 3):S135-37

20. Aime S, Caravan P. Biodistribution of gadolinium-based contrast agents, including gadolinium deposition. J Magn Reson Imaging 2009;30:1259-67

21. Hergan K, Doringer W, Längle M, et al. Effects of iodinated contrast agents in MR imaging. Eur J Radiol 1995;21:11-17

22. Algin $\mathrm{O}$, Turkbey B. Intrathecal gadolinium-enhanced MR cisternography: a comprehensive review. AJNR Am J Neuroradiol 2013;34:14-22

23. Park KW, Im SB, Kim BT, et al. Neurotoxic manifestations of an overdose intrathecal injection of gadopentetate dimeglumine. $J \mathrm{Ko}$ rean Med Sci 2010;25:505-08

24. Wendl CM, Schambach F, Zimmer C, et al. CT myelography for the planning and guidance of targeted epidural blood patches in patients with persistent spinal CSF leakage. AJNR Am J Neuroradiol 2012;33:541-44 